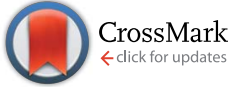

Cite this: RSC Adv., 2017, 7, 2351

Received 3rd November 2016 Accepted 15th December 2016

DOI: $10.1039 / c 6 r a 26267 h$

www.rsc.org/advances

\section{In situ redox deposition of palladium nanoparticles on oxygen-deficient tungsten oxide as efficient hydrogenation catalysts $\uparrow$}

\author{
Na Xue, ${ }^{a}$ Rui-Jin Yu, ${ }^{\text {*a }}$ Cheng-Zong Yuan, ${ }^{\text {b } X i a o ~ X i e, ~}{ }^{\text {b }}$ Yi-Fan Jiang, ${ }^{\mathrm{b}}$ Hong-Yan Zhou, ${ }^{\mathrm{c}}$ \\ Tuck-Yun Cheang*c and An-Wu Xu*b
}

\begin{abstract}
Noble metal/metal oxide support hybrid materials have attracted tremendous interest due to their wide applications in catalysis. Herein, we have developed a novel and surfactant-free method to prepare $\mathrm{Pd} /$ $\mathrm{WO}_{3-x}$ composite materials with clean surfaces. Oxygen-vacancy-rich $\mathrm{WO}_{3-x}$ nanowires (NWs) provide free electrons to reduce $\mathrm{Pd}^{2+}$, and surface-clean $\mathrm{Pd}$ nanoparticles (NPs) directly grow on $\mathrm{WO}_{3-x}$ surfaces through an in situ redox reaction between reductive $\mathrm{WO}_{3-x}$ and metal salt precursor $\left(\mathrm{Na}_{2} \mathrm{PdCl}_{4}\right)$ in aqueous solution. The as-obtained $\mathrm{Pd} / \mathrm{WO}_{3-x}$ nanocomposites show excellent catalytic activities for the hydrogenation of 4-nitrophenol (4-NP) and styrene. The apparent rate constant for 4-NP reduction is $0.045 \mathrm{~s}^{-1}$, over the $\mathrm{Pd} / \mathrm{WO}_{3-x}$ catalyst. The turnover frequency (TOF) value for styrene hydrogenation is $1074.5 \mathrm{~h}^{-1}$, thus, exhibiting high catalytic performance. Moreover, the obtained $\mathrm{Pd} / \mathrm{WO}_{3-x}$ catalyst exhibits good stability. Oxygen vacancies in $\mathrm{WO}_{3-x} \mathrm{NWs}$ can accelerate electron transport and promote hydrogen adsorption and dissociation on the surface of the catalyst. The strong interaction between Pd NPs and $\mathrm{WO}_{3-x}$ support contributes to the excellent performance. Our work provides a novel and simple strategy to directly fabricate other-noble metal NP loaded oxygen-deficient metal oxides as highly efficient catalysts for chemical transformation.
\end{abstract}

\section{Introduction}

The fabrication of noble metal/semiconductor metal oxide hybrid materials has aroused much attention because of their characteristic catalytic, optical and electrical properties. ${ }^{1,2}$ Among them, tungsten oxide-based materials have been widely studied due to potential applications such as gas sensors, ${ }^{3}$ photocatalysts, ${ }^{4}$ electrochromic devices ${ }^{5}$ and field-emission devices. ${ }^{6}$ Tungsten oxide is well documented for its nonstoichiometric character, where the crystal lattice can withstand a large number of oxygen vacancies. ${ }^{7,8}$ In particular, the presence of oxygen vacancies in tungsten oxide $\left(\mathrm{WO}_{3-x}, x>0\right)$ receives considerable attention because of its unusual defect structure and unique characteristics. The existence of oxygen vacancies is reported to enhance catalytic activities by

${ }^{a}$ College of Science, Northwest A\&F University, Yangling, Shaanxi 712100, P. R. China. E-mail: yuruijin@nwsuaf.edu.cn

${ }^{b}$ Division of Nanomaterials and Chemistry, Hefei National Laboratory for Physical Sciences at Microscale, University of Science and Technology of China, Hefei 230026, China. E-mail: anwuxu@ustc.edu.cn

${ }^{C}$ Department of Vascular Surgery, Department of Neurological Intensive Care Unit, The First Affiliated Hospital of Sun Yat-Sen University, Guangzhou, 510080, P. R. China. E-mail: 13631322559@163.com

$\dagger$ Electronic supplementary information (ESI) available: XRD patterns, SEM images, TEM images, HRTEM images, and UV-visible absorption spectra. See DOI: $10.1039 / \mathrm{c} 6 \mathrm{ra} 26267 \mathrm{~h}$ narrowing the band gap and providing additional active catalytic sites. ${ }^{9}$ As a result, oxygen-deficient $\mathrm{WO}_{3-x}$ often displays critical impact on its physicochemical properties such as tuning optical absorption and increasing conductivity.

Moreover, recent reports have manifested that noble metal nanoparticle (NPs) loaded supports can function as effective catalysts in chemical and photochemical reactions. ${ }^{10}$ However, a serious problem in the application of supported catalysts is the agglomeration of noble metal NPs. Collectively, many researchers offer control over basic parameters such as particle size and shape to further enhance their properties by the passivation of specific surfactant or ligand molecules. These stabilizing molecules can be successfully removed by thermal and oxidative approaches, which usually requires a high temperature and often inevitably leads to an increase in the size of metal NPs. ${ }^{11}$ It has already been proved beyond doubt that catalysis involving noble metal NPs depends on the size and the surface of the catalyst particle. ${ }^{12}$ Therefore, direct deposition of noble metal NPs on oxygen-defective semiconductor via a facile method without any stabilizing agents is highly desired.

Surface oxygen vacancy sites are proved to have the reductive ability for the reduction of metal ions. Pan and co-workers reported the synthesis of $\mathrm{TiO}_{2}$ with oxygen vacancies by a photocatalytic reaction, and noble-metal NPs (Ag, Pt, and Pd) can grow directly on $\mathrm{TiO}_{2}$ surfaces via a redox reaction between reductive $\mathrm{TiO}_{2}$ and metal salt precursors without any stabilizing 
agents or reducing agents. ${ }^{\mathbf{1 3}}$ The obtained $\mathbf{M}-\mathrm{TiO}_{2}$ nanocomposites exhibit the enhancement in photocatalytic activities toward selective oxidation of benzyl alcohol and reduction of heavy metal $\mathrm{Cr}(\mathrm{vI})$ ions under visible light irradiation. $\mathrm{Hu}$ and co-workers prepared $\mathrm{Au} / \mathrm{TiO}_{2}$ nanocomposites through an in situ self-redox reaction between oxygen-defect-rich $\mathrm{TiO}_{2-x}$ and $\mathrm{HAuCl}_{4}$ in aqueous solution. ${ }^{\mathbf{1 4}}$ This process is named as a selfredox process without the use of any reducing agents or surfactant molecules. The $\mathrm{Au}$ loaded $\mathrm{TiO}_{2}$ nanosheets display greatly enhanced photocatalytic performance in the photocatalytic degradation of toxic pollutants.

There are few reports on the preparation of tungsten oxide nanostructures with oxygen vacancies and noble metal NPs directly grow on tungsten oxide surfaces. Herein, we report a novel and simple route for synthesis of $\mathrm{WO}_{3-x}$ single crystal NWs with abundant oxygen vacancies. The main focus of this work is to find suitable conditions for an effective introduction of oxygen vacancies into metal oxides. It is reported that vacuum or hydrogen treatment can induce the surface disorder of metal oxides. ${ }^{15,16}$ Here, we develop a surfactant-free way to obtain oxygen-vacancy-rich $\mathrm{WO}_{3-x} \mathrm{NWs}$ by one-step hydrothermal route. More importantly, it was reported that non-stoichiometric $\mathrm{WO}_{3-x}$ is thermodynamically stable at room temperature, and the quenching of oxygen vacancies was found to occur only upon annealing in air at a temperature higher than $400{ }^{\circ} \mathrm{C} .{ }^{17} \mathrm{Pd}$ NPs grow directly on $\mathrm{WO}_{3-x}$ surfaces through an in situ redox reaction between reductive $\mathrm{WO}_{3-x}$ and metal salt precursor $\left(\mathrm{Na}_{2} \mathrm{PdCl}_{4}\right)$ in aqueous solution, and strong metal-support interaction occurs. The resulting $\mathrm{Pd} / \mathrm{WO}_{3-x}$ nanocatalyst is used as catalyst for hydrogenation reactions such as the reduction of nitro-aromatic compounds to amines and the hydrogenation of styrene.

\section{Experimental}

\subsection{Materials}

$\mathrm{Na}_{2} \mathrm{PdCl}_{4}$ was purchased from Alfa Aesar. Ammonium tungstate hydrate, citric acid and $\mathrm{NaBH}_{4}$ were obtained from Sinopharm Chemical Reagent Co., Ltd. All chemicals were used as received without further purification. Double distilled water was used in all experiments.

\subsection{Preparation}

To synthesize $\mathrm{WO}_{3-x}$ nanowires, $0.913 \mathrm{~g}$ of ammonium tungstate hydrate $(0.3 \mathrm{mmol})$ and $0.183 \mathrm{~g}$ of citric acid were dissolved in distilled water under stirring. Subsequently, the mixture was transferred to a $100 \mathrm{~mL}$ Teflon liner and then heated at $160{ }^{\circ} \mathrm{C}$ for $12 \mathrm{~h}$. After cooling to room temperature naturally, the deepblue precipitates were collected and washed with distilled water and ethanol several times. The final products were dried in vacuum at $60{ }^{\circ} \mathrm{C}$ overnight. In the loading process, a facile method was used to load the palladium particles for direct growth on $\mathrm{WO}_{3-x}$ nanowires in situ. The synthesized $\mathrm{WO}_{3-x}$ $(0.100 \mathrm{~g})$ was dispersed in distilled water $(20 \mathrm{~mL})$, and an amount of $\mathrm{Na}_{2} \mathrm{PdCl}_{4}(10 \mathrm{mM})$ was added dropwise with constant stirring at room temperature overnight. Finally, the deep-gray solid was collected and dried under vacuum at $60{ }^{\circ} \mathrm{C}$ overnight.

\subsection{Catalyst characterization}

$\mathrm{X}$-ray diffraction (XRD) patterns were recorded using a Rigaku/ Max-3A X-ray diffractometer with $\mathrm{Cu} \mathrm{K} \alpha$ radiation $(\lambda=$ $1.54178 \AA$ A). Field emission scanning electron microscopy observations (FE-SEM) were obtained using a JEOLJSM-6300F field-emission scanning electron microscope (15 kV). The transmission electron microscopy (TEM) images, high resolution transmission electron microscopy (HRTEM) images and elemental mapping were gathered on a JEOL JEM12100F field emission high resolution transmission electron microscope operated at $200 \mathrm{kV}$. X-ray photoelectron spectroscopy (XPS) measurements was done on a Perkin-Elmer RBD upgraded PHI5000C ESCA system. The actual content of Pd was measured with a Thermo Scientific Plasma Quad 3 inductively-coupled plasma mass spectrometry (ICP-MS) after dissolving sample with a mixture of $\mathrm{HCl}$ and $\mathrm{HNO}_{3}(3: 1$, volume ratio). Raman spectra were obtained at room temperature with a Perkin-Elmer 400F Raman spectrometer using a $514.5 \mathrm{~nm}$ laser beam. A Shimadzu spectrophotometer (Model 2501 PC) was used to record the UV-vis diffuse reflectance spectra of the samples in the region of 200 to $800 \mathrm{~nm}$. The electron paramagnetic resonance (EPR) spectra were recorded on a JEOL JESFA200 EPR spectrometer (298 K, $9061 \mathrm{MHz}, 0.998 \mathrm{~mW}$, X-band).

\subsection{Catalytic reduction of 4-nitrophenol}

The catalytic properties of $\mathrm{Pd} / \mathrm{WO}_{3-x}$ (Pd, 0.99 wt\%) nanocomposites were investigated via the reduction of 4-nitrophenol to 4-aminophenol with $\mathrm{NaBH}_{4}$ as the reductant under ambient temperature as a model reaction. First, 4-nitrophenol $(150 \mu \mathrm{L}$, $10 \mathrm{mmol} \mathrm{L}^{-1}$ ) was added to $\mathrm{NaBH}_{4}$ aqueous solution $(8 \mathrm{~mL}$, $0.1 \mathrm{~mol} \mathrm{~L}^{-1}$ ), and the mixture was stirred for several minutes. Then $\mathrm{Pd} / \mathrm{WO}_{3-x}$ nanocomposites aqueous suspension $(50 \mu \mathrm{L}$, $2.8 \mathrm{mg} \mathrm{mL}^{-1}$ ) was added to the above solution, which was stirred until the bright yellow gradually changed to colorless. The reaction progress was monitored by measuring the UV-vis absorption spectra of the reaction solutions.

\subsection{Catalytic hydrogenation of styrene}

$\mathrm{Pd} / \mathrm{WO}_{3-x}(5.5 \mathrm{mg})$, styrene $(570 \mu \mathrm{L}, 5.0 \mathrm{mmol})$ and 1,3,5-trimethylbenzene (internal standard, $500 \mu \mathrm{L}, 3.6 \mathrm{mmol}$ ) were added into $10 \mathrm{~mL}$ ethanol. The air in the reactor was replaced by $\mathrm{H}_{2}$. Then, the mixture was stirred in room temperature (about $30{ }^{\circ} \mathrm{C}$ ) for the desired time. The product was analyzed by a gas chromatograph (GC) equipped with a flame ionization detector (FID). Gas chromatograms (GC) were obtained on Agilent HP-5, Gas Chromatograph with a SGE BP1 non-polar 100\% dimethylpolysiloxane capillary column of $(30 \mathrm{~m} \times 0.32 \mathrm{~mm} \times 0.25$ $\mu \mathrm{m})$ dimensions. The method used for styrene hydrogenation is performed for 1 minute at $70{ }^{\circ} \mathrm{C}$ and a ramp of $10{ }^{\circ} \mathrm{C} \mathrm{min}{ }^{-1}$ until $110{ }^{\circ} \mathrm{C}$.

\section{Results and discussion}

The synthetic procedure of $\mathrm{Pd} / \mathrm{WO}_{3-x}$ nanocatalyst is elucidated in Fig. 1. In the first step, $\mathrm{WO}_{3-x}$ nanowires (NWs) with abundant oxygen vacancies were synthesized from ammonium 


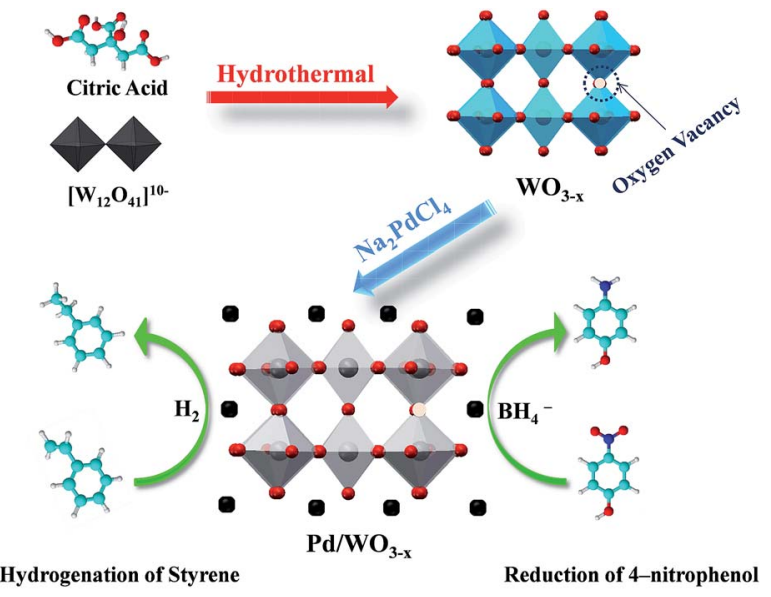

- Pd OW

Fig. 1 Schematic illustration for the synthesis of oxygen-vacancy-rich $\mathrm{WO}_{3-x} \mathrm{NWs}$ and $\mathrm{Pd} / \mathrm{WO}_{3-x}$ nanocomposites. The resulting $\mathrm{Pd} / \mathrm{WO}_{3-x}$ nanocatalyst is used for hydrogenation reactions of 4-nitrophenol (4NP) and styrene.

tungstate hydrate and citric acid by a one-step hydrothermal treatment. Subsequently, the as-synthesized $\mathrm{WO}_{3-x} \mathrm{NWs}$ and $\mathrm{Na}_{2} \mathrm{PdCl}_{4}$ were mixed in aqueous solution by stirring, $\mathrm{Pd}^{2+}$ ions were directly reduced by oxygen-vacancy-rich $\mathrm{WO}_{3-x} \mathrm{NWs}$, and surface-clean palladium nanoparticles (NPs) were deposited on $\mathrm{WO}_{3-x}$ surfaces at room temperature through an in situ redox reaction. The obtained $\mathrm{Pd} / \mathrm{WO}_{3-x}$ nanocatalyst was used to evaluate catalytic performance of the hydrogenation of styrene and 4-nitrophenol (see Experimental section).

The X-ray diffraction (XRD) analysis was performed to examine the crystalline structure of obtained $\mathrm{WO}_{3-x} \mathrm{NWs}$ and $\mathrm{Pd} / \mathrm{WO}_{3-x}$, and the results are shown in Fig. 2a and $\mathrm{S} 1 . \dagger$ The XRD pattern displays a pure phase of hexagonal crystalline structure with diffraction peaks assigned to tungsten trioxide (JCPDS no. 85-2459). The typical lattice constants were calculated to be $a=b=7.326 \AA, c=7.669 \AA$, confirming that $\mathrm{WO}_{3-x}$ was successfully synthesized by hydrothermal method. The diffraction peaks of Pd were not observed for $\mathrm{Pd} / \mathrm{WO}_{3-x}$ (Fig. 2a), likely due to its low weight content (2.3 wt\% Pd) by inductively coupled plasma-atomic emission spectroscopy (ICPAES) analysis.

The morphology and structural features of as-synthesized $\mathrm{WO}_{3-x}$ NWs were analyzed by scanning electron microscopy (SEM) and transmission electron microscopy (TEM). The SEM images shown in Fig. $2 \mathrm{~b}$ and $\mathrm{S} 2, \dagger$ reveal $\mathrm{WO}_{3-x}$ NWs are uniform and very long. As estimated from the SEM image, the obtained $\mathrm{WO}_{3-x} \mathrm{NWs}$ are 15-20 nm in diameters and 0.05-1.5 $\mu \mathrm{m}$ in lengths. TEM image further shows that the obtained $\mathrm{WO}_{3-x}$ is composed of a large number of radial NWs (Fig. S3a, ESI $\dagger$ ). It is clearly seen from TEM images that the surfaces of $\mathrm{WO}_{3-x}$ NWs are decorated with Pd NPs (Fig. S3b, ESI $\dagger$ ).

Clear lattice fringes measured with an interplanar lattice spacing of $0.367 \mathrm{~nm}$ for $\mathrm{WO}_{3-x}$ and $0.242 \mathrm{~nm}$ for Pd NPs can be unambiguously observed from the high-resolution TEM (HRTEM) images (Fig. 2c), which corresponds to the (110)
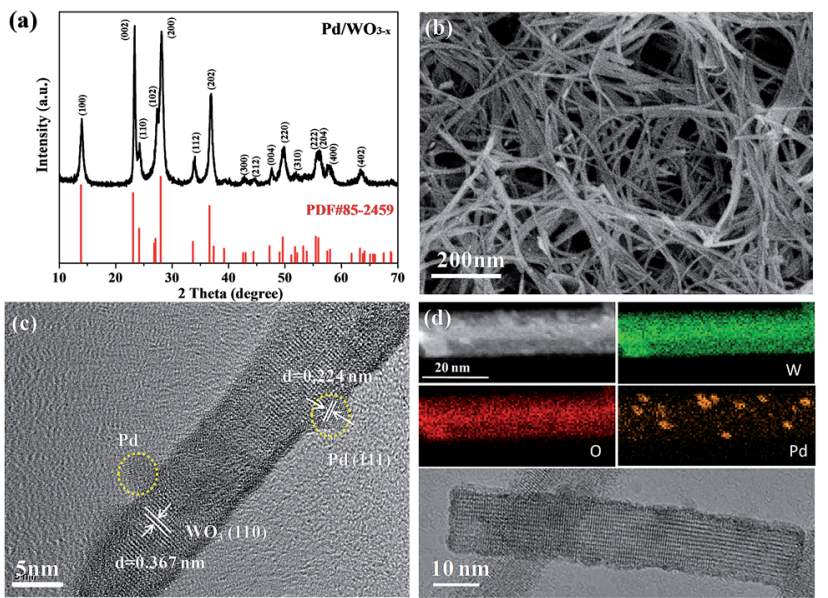

Fig. 2 (a) XRD pattern of the as-prepared $\mathrm{Pd} / \mathrm{WO}_{3-x}$. The standard card for hexagonal $\mathrm{WO}_{3}$ (JCPDS no. 85-2459) is shown at the bottom. (b) SEM image of $\mathrm{WO}_{3-x} \mathrm{NWs}$. (c) HRTEM image of $\mathrm{Pd} / \mathrm{WO}_{3-x}$ nanocomposites. (d) STEM image and the corresponding elemental mappings of $\mathrm{Pd} / \mathrm{WO}_{3-x}$ (actual weight content of $\mathrm{Pd}, 2.3 \mathrm{wt} \%$ ).

atomic plane of tungsten oxide and the (111) crystal plane of palladium, respectively. Simultaneously, the HRTEM images of $\mathrm{WO}_{3-x}$ also exhibit a wires-like morphology with an interfacial angle of $90^{\circ}$ between (110) and (1-10) facets (Fig. S4, ESI $\dagger$ ), corresponding to the lattice space of $0.367 \mathrm{~nm}$ and $0.320 \mathrm{~nm}$, respectively. From these results, it is concluded that each hexagonal $\mathrm{WO}_{3-x}$ nanowire grew preferentially along the [110] direction. Meanwhile, the TEM and HRTEM images clearly exhibit that Pd NPs were well-dispersed on $\mathrm{WO}_{3-x}$ support with a narrow size distribution in the range of $2-5 \mathrm{~nm}$. As expected, elemental mapping analysis (Fig. 2d) of the as-obtained Pd/ $\mathrm{WO}_{3-x}$ composites clearly displays $\mathrm{W}, \mathrm{O}$ and Pd elementals, confirming that the surfaces of $\mathrm{WO}_{3-x}$ NWs are decorated with small Pd NPs.

The compositions and valence states of $\mathrm{WO}_{3-x}$ NWs and $\mathrm{Pd} /$ $\mathrm{WO}_{3-x}$ were investigated by X-ray photoelectron spectroscopy (XPS) measurements (Fig. 3). For $\mathrm{WO}_{3-x} \mathrm{NWs}$, the $\mathrm{W}$ 4f region exhibits two peaks at $37.5 \mathrm{eV}$ of and $35.4 \mathrm{eV}$, which are attributed to $\mathrm{W}^{6+}$. The binding energies appeared at $36.5 \mathrm{eV}$ and $34.6 \mathrm{eV}$ are assigned to the lower oxidation state $\mathrm{W}^{5+}$ (Fig. 3a). ${ }^{18,19} \mathrm{~W}$ if peaks for $\mathrm{Pd} / \mathrm{WO}_{3-x}$ sample shift to lower binding energies as compared to bare $\mathrm{WO}_{3-x}$ (Fig. $3 \mathrm{~b}$ ) due to free electrons in oxygen vacancy abundant $\mathrm{WO}_{3-x}$ transfer to Pd. This result suggests the strong interaction occurs between Pd NPs and $\mathrm{WO}_{3-x} \mathrm{NWs}^{20}$ The XPS results clearly demonstrate that $\mathrm{W}^{5+}$ was introduced into the final sample, consequently, oxygen vacancies were formed in $\mathrm{WO}_{3-x} \mathrm{NWs}$, which might have an effect on the catalytic activity. The content of $\mathrm{W}^{5+}$ was estimated to be about $16.5 \%$ in $\mathrm{WO}_{3-x}$ sample from XPS data. After the deposition of Pd NPs, the relative content of $\mathrm{W}^{5+}$ was reduced to about $11.9 \%$ from XPS analysis (Fig. S5, ESI $\dagger$ ), suggesting $\mathrm{Pd}^{2+}$ ions were reduced by $\mathrm{W}^{5+}$ centers.

It is noted that the color of $\mathrm{WO}_{3-x}$ aqueous suspension changed from original blue to gray after adding a given concentration of $\mathrm{Na}_{2} \mathrm{PdCl}_{4}$ (Fig. S6, ESI $\dagger$ ). This change in the color is attributed to the partial disappearance of oxygen 

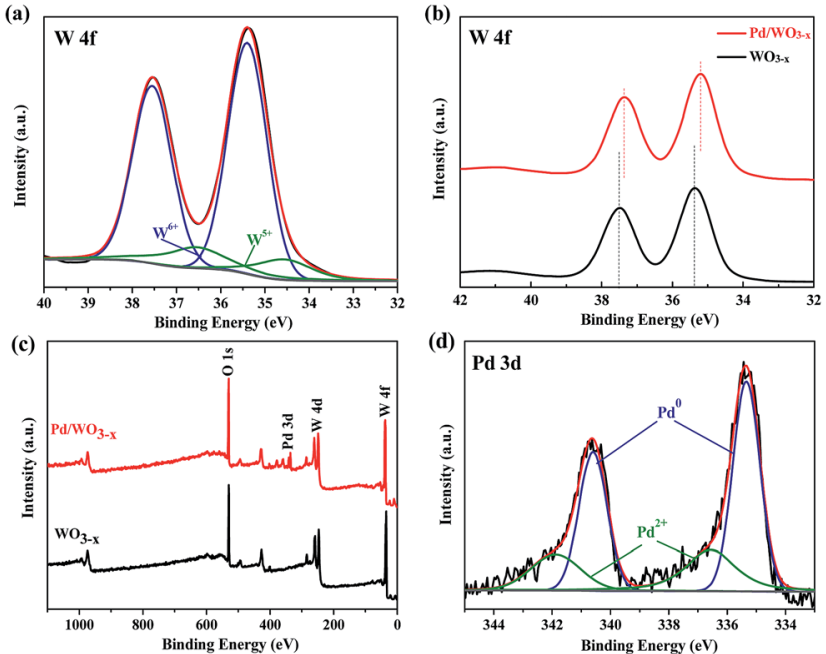

Fig. 3 (a) The $\mathrm{W} 4 \mathrm{f}$ spectrum of $\mathrm{WO}_{3-x} \mathrm{NWs}$. (b) The $\mathrm{W} 4 \mathrm{f}$ spectrum of $\mathrm{Pd} / \mathrm{WO}_{3-x}$ composites (red line) and $\mathrm{WO}_{3-x}$ (black line). (c) XPS survey scan spectra of $\mathrm{WO}_{3-x} \mathrm{NWs}$ and $\mathrm{Pd} / \mathrm{WO}_{3-x}(\mathrm{Pd}, 0.99 \mathrm{wt} \%)$ sample. (d) The $\mathrm{Pd} 3 \mathrm{~d}$ spectrum of $\mathrm{Pd} / \mathrm{WO}_{3-x}$ sample.

vacancies and the formation of Pd NPs because oxygen vacancies provide free electrons for the reduction of $\mathrm{Pd}^{2+}$ ions. Furthermore, the color of the obtained $\mathrm{WO}_{3-x}$ had no significant change after at least six months, indicating that $\mathrm{WO}_{3-x}$ is stable in air. As show in Fig. 3c, XPS survey spectrum of Pd/ $\mathrm{WO}_{3-x}$ sample clearly displays the presence of $\mathrm{W}, \mathrm{O}$ and $\mathrm{Pd}$ elements, indicating the formation of $\mathrm{Pd} / \mathrm{WO}_{3-x}$. For highresolution Pd 3d XPS spectrum of $\mathrm{Pd} / \mathrm{WO}_{3-x}$ (Fig. 3d), it can be seen that two peaks with binding energies of $335.3 \mathrm{eV}$ and $340.6 \mathrm{eV}$ are ascribed to $\mathrm{Pd} 3 \mathrm{~d}_{5 / 2}$ and $\mathrm{Pd} 3 \mathrm{~d}_{3 / 2}$ of $\mathrm{Pd}^{0}$, respectively. ${ }^{21,22}$ Moreover, the other two weak peaks appeared at $336.6 \mathrm{eV}$ and $341.9 \mathrm{eV}$ could be assigned to $\mathrm{Pd}^{2+}$. The XPS results clearly reveal that most of $\mathrm{Pd}^{2+}$ ions were reduced to $\mathrm{Pd}^{0}$ by oxygen-vacancy-rich $\mathrm{WO}_{3-x} \mathrm{NWs}$. Notably, the binding energies of Pd 3d signals for $\mathrm{Pd} / \mathrm{WO}_{3-x}$ sample has a positive-shift by about $0.3 \mathrm{eV}$ compared to the standard peak position. ${ }^{23}$ This shift in binding energies is influenced by the electronic modification of $\mathrm{Pd}$ by $\mathrm{WO}_{3-x}$ support and the size of metal particles, ${ }^{24}$ indicating strong metal-support interaction (SMSI) occurs in $\mathrm{Pd} / \mathrm{WO}_{3-x}$, which leads to the enhanced catalytic performance of the obtained catalyst.

To further verify the formation of oxygen vacancies, photophysical properties were characterized by UV-visible diffuse reflectance spectra (Fig. 4a). The commercial $\mathrm{WO}_{3}$ only responds to visible light due to its intrinsic band gap $(2.6 \mathrm{eV}$, Fig. 4b). For as-synthesized $\mathrm{WO}_{3-x} \mathrm{NWs}$, the strong absorption in the visible and near infrared (NIR) regions is clearly observed, thus providing clear evidence that the non-stoichiometric $\mathrm{WO}_{3-x}$ NWs contain a large number of oxygen vacancies. ${ }^{25,26}$ Moreover, in contrast to commercial $\mathrm{WO}_{3}$ which appears as yellowish powders, the color of obtained $\mathrm{WO}_{3-x}$ NWs is deep blue because of the presence of abundant oxygen vacancies. ${ }^{27}$ Raman spectroscopy was further performed to analyze the crystalline structure of $\mathrm{WO}_{3-x} \mathrm{NWs}$, which is sensitive to the surface oxygen vacancies (Fig. 4c). Raman spectrum of
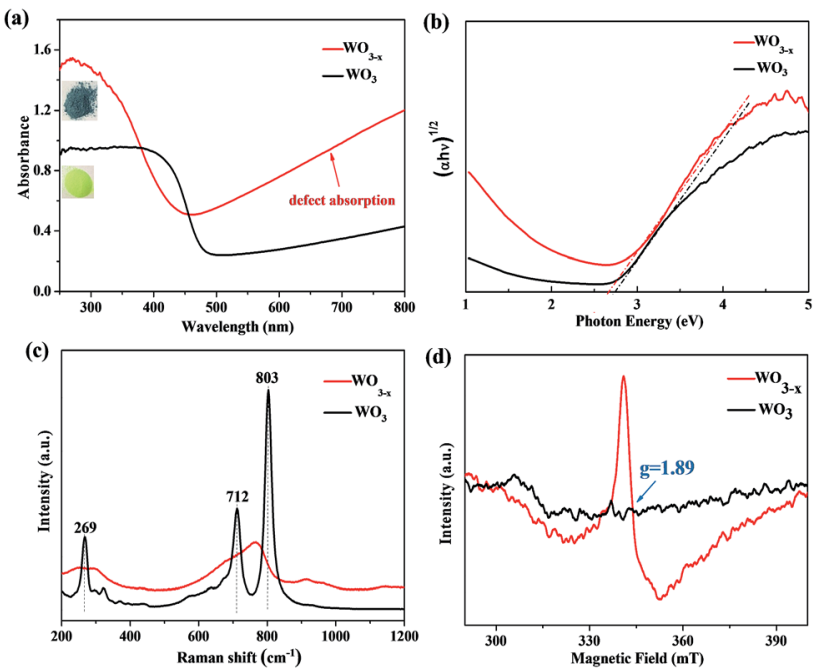

Fig. 4 (a) The UV-visible diffuse reflectance spectra for $\mathrm{WO}_{3-x} \mathrm{NWs}$ (red line) and commercial $\mathrm{WO}_{3}$ (black line). The insets in (a) show the corresponding photographs. (b) Curves of the Kubelka-Munk function plotted against the photon energy for $\mathrm{WO}_{3-x}$ and $\mathrm{WO}_{3}$. (c) Raman spectra of commercial $\mathrm{WO}_{3}$ and as-synthesized $\mathrm{WO}_{3-x} \mathrm{NWs}$. (d) The $\mathrm{X}$-band EPR spectra of deep blue $\mathrm{WO}_{3-x} \mathrm{NWs}$ and commercial $\mathrm{WO}_{3}$ as a reference sample recorded at $T=298 \mathrm{~K}$.

commercial $\mathrm{WO}_{3}$ is dominated by three characteristic vibration bands at 269, 712, and $803 \mathrm{~cm}^{-1}$, corresponding to bending vibration of $\delta(\mathrm{O}-\mathrm{W}-\mathrm{O})$ and the stretching vibration of $\nu(\mathrm{W}-\mathrm{O}-$ W). ${ }^{28}$ For $\mathrm{WO}_{3-x}$ NWs, Raman peaks are much broader and lower intensity as compared to commercial $\mathrm{WO}_{3}$. The broad characteristic peaks centered in the region of $200-400 \mathrm{~cm}^{-1}$ and $600-900 \mathrm{~cm}^{-1}$ result from the wide range of $\mathrm{W}-\mathrm{O}-\mathrm{W}$ bond lengths. This change is identified by the existence of $\mathrm{W}^{5+}-\mathrm{O}$ vibrations in $\mathrm{WO}_{3-x}$ sample, ${ }^{\mathbf{1 8 , 2 9}}$ which is expected to increase the concentration of oxygen vacancies. To explore $\mathrm{W}^{5+}$ states in $\mathrm{WO}_{3-x}$ sample, we measured electron paramagnetic resonance (EPR) spectra performed at $298 \mathrm{~K}$. As shown in Fig. 4d, a strong EPR signal observed at $g=1.89$ is assigned to $\mathrm{W}^{5+},{ }^{30,31}$ which is in agreement with the UV-vis spectra and XPS analysis. Interestingly, the EPR signal shows a significant decline after the deposition of Pd NPs (Fig. S7, ESI $\dagger$ ), which indicates the concentration of oxygen vacancies decreases in $\mathrm{WO}_{3-x} \mathrm{NWs}$. These results, taken together, demonstrate the successful introduction of oxygen vacancies into tungsten oxide and in situ redox deposition of $\mathrm{Pd}$ onto $\mathrm{WO}_{3-x}$ surface with a strong interaction.

The reduction of nitro-aromatic compounds to amines is a significant process in synthetic organic chemistry of manufacturing many potent industrial intermediates. ${ }^{32}$ More recently, noble metal NPs like $\mathrm{Au}, \mathrm{Ag}$ and Pd have been investigated to catalyze the well-known model reduction of 4-nitrophenol (4-NP) to 4-aminophenol (4-AP) owing to their efficient catalytic performance. ${ }^{33,34}$ Here, the catalytic activity of obtained $\mathrm{Pd} / \mathrm{WO}_{3-x} \mathrm{NWs}$ was evaluated by the reduction of 4-NP to 4-AP in the presence of hydrogen source, $\mathrm{NaBH}_{4}$. When the reaction mixture contained 4-NP and $\mathrm{NaBH}_{4}$, the change in concentration was monitored by UV-vis absorption spectroscopy as 
a function of time (Fig. 5). After adding an amount of $\mathrm{NaBH}_{4}$ solution into 4-NP, the aqueous 4-NP solution undergone an immediate red-shift in the UV-vis absorbance band from 317 to $400 \mathrm{~nm}$ (Fig. S8a, ESI $\dagger$ ), the change of color from light yellow to deep yellow could be attributed to the formation of 4-nitrophenolate ion. ${ }^{32,35}$ The intensity of the absorption peak of 4-NP at $400 \mathrm{~nm}$ was not reduced within $30 \mathrm{~min}$ in the presence of $\mathrm{NaBH}_{4}$ without catalyst (Fig. S8b, ESI†). This result indicates that it is difficult for this reaction to proceed in the absence of catalyst. When a small amount of $\mathrm{Pd} / \mathrm{WO}_{3-x}$ catalyst was added into the reaction system, the absorbance peak of $4-\mathrm{NP}$ at $400 \mathrm{~nm}$ rapidly decreased, suggesting the successive reduction of 4-NP. Meanwhile, a concomitant increased peak of 4-AP at $298 \mathrm{~nm}$ was observed, thus confirming the transformation from 4-NP to 4-AP (Fig. 5a). It is noted that, when $\mathrm{Pd} / \mathrm{WO}_{3-x}$ was added to the reaction system, the solution color quickly turned from deep yellow to colorless within $80 \mathrm{~s}$. In addition, the rate constant $k$, calculated by the linear relationship between $\ln \left(C_{t} / C_{0}\right)$ and reaction time (Fig. $5 \mathrm{~b}$ ) for 4-NP reduction, was found to be 0.045 $\mathrm{s}^{-1}$, corresponding to the first-order reaction kinetics. Additionally, bare $\mathrm{WO}_{3-x}$ NWs was also used to test the reaction under the same conditions. Almost no reaction was detected by monitoring UV-vis absorption (Fig. 5b), indicating that it is noble metal that effectively promotes the catalytic activity. The activity of our catalyst is comparable to those reported in the literature, ${ }^{36-38}$ as shown in Table S1 (ESI $\dagger$ ).

The catalytic mechanism of the reduction of 4-NP is proposed in Scheme 1. $\mathrm{NaBH}_{4}$ can react with water at room temperature to yield $\mathrm{H}_{2}$, then, $\mathrm{Pd} / \mathrm{WO}_{3-x}$ catalyst dissociates hydrogen, and Pd NPs act as electron relay system to transfer electrons donated by $\mathrm{BH}_{4}{ }^{-}$to the nitro groups of $4-\mathrm{NP}$, as a result, the nitro group was reduced to the nitroso group (I). Followed by formation of hydroxylamine (II) and then hydroxylamine was further reduced to the amino group (III) with the dehydration process, leading to the final 4-AP product. ${ }^{39}$

To further investigate the catalytic activity of the resulting $\mathrm{Pd} / \mathrm{WO}_{3-x}$ nanocomposites, the catalytic hydrogenation of styrene was selected and carried out at room temperature and 1 atm $\mathrm{H}_{2}$. In terms of the hydrogenation of styrene, the noble metal based catalysts display a remarkable catalytic activity toward the hydrogenation of styrene. ${ }^{40,41}$ In this reaction,
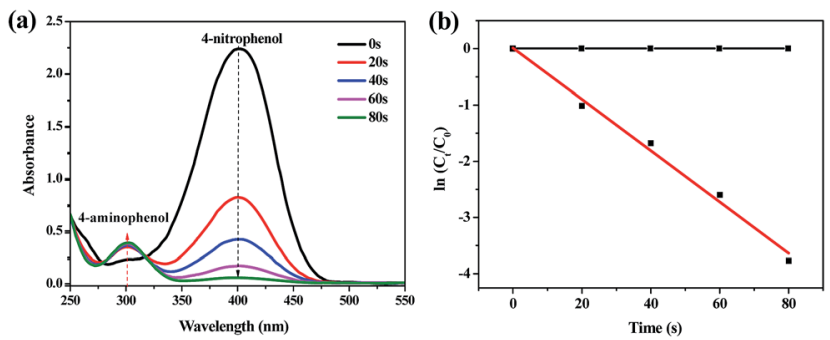

Fig. 5 (a) UV-visible absorption spectra of 4-NP reduced by $\mathrm{NaBH}_{4}$ with $\mathrm{Pd} / \mathrm{WO}_{3-x}$ catalyst at room temperature. (b) Kinetic curves for the reduction of 4-NP catalyzed by $\mathrm{Pd} / \mathrm{WO}_{3-x}$ nanocatalyst (red line) and bare $\mathrm{WO}_{3-x} \mathrm{NWs}$ (black line), respectively. $C_{t}$ and $C_{0}$ are 4-NP concentrations at time $t$ and 0 , respectively.

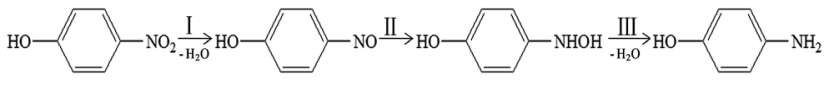

Scheme 1 A plausible mechanism for the reduction of 4-NP by $\mathrm{NaBH}_{4}$ over $\mathrm{Pd} / \mathrm{WO}_{3-x}$ catalyst.

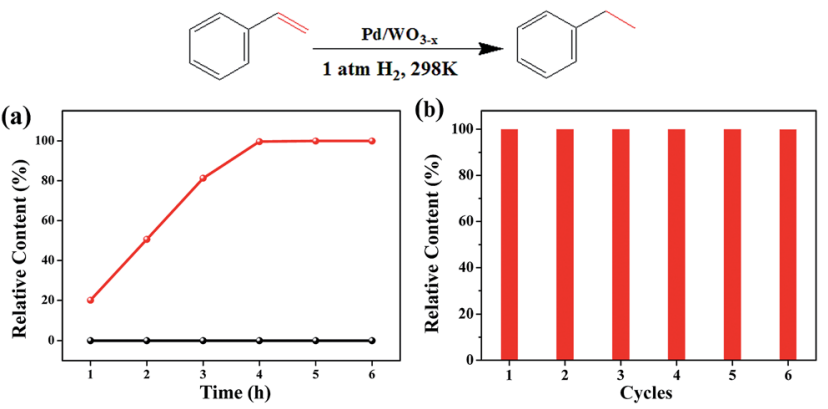

Fig. 6 (a) Typical conversion-time profile of ethylbenzene product from styrene hydrogenation over $\mathrm{Pd} / \mathrm{WO}_{3-x}$ catalyst (red line) and $\mathrm{WO}_{3-x} \mathrm{NWs}$ (black line), respectively. (b) Bar diagram of recycling ability of $\mathrm{Pd} / \mathrm{WO}_{3-x}$ catalyst for hydrogenation reaction of styrene.

styrene is readily converted into ethylbenzene over catalyst by using hydrogen gas as the hydrogen source. Fig. 6a shows the relationship between conversion of styrene and time by using $\mathrm{Pd} / \mathrm{WO}_{3-x}(\mathrm{Pd}, 2.3 \mathrm{wt} \%$ ) catalyst. After 4 hours of reaction, the catalyst converted more than $99.5 \%$ of styrene to ethylbenzene without any detectable byproducts. Furthermore, we also evaluated the catalytic activity of bare $\mathrm{WO}_{3-x} \mathrm{NWs}$ in the absence of noble metal. The result shows that almost no ethylbenzene was detected, indicating that it is difficult for the hydrogenation reaction to proceed without noble metal. The turnover frequency (TOF) value of $\mathrm{Pd} / \mathrm{WO}_{3-x}$ catalyst, defined as the number of moles of substrate converted per mol of palladium per hour, was calculated to be $1074.5 \mathrm{~h}^{-1}$. The catalytic efficiency or TOF value of our $\mathrm{Pd} / \mathrm{WO}_{3-x}$ nanocatalyst is comparable to other previously reported Pd based catalysts, ${ }^{42,43}$ thus displaying a high activity. The reusability of $\mathrm{Pd} / \mathrm{WO}_{3-x}$ nanocatalyst in repeated catalytic reaction was performed in six consecutive runs (Fig. 6b). The results exhibit that the catalyst successfully recycled at least 6 times without obvious loss in activity. The high catalytic performance of $\mathrm{Pd} / \mathrm{WO}_{3-x}$ nanocatalyst is probably attributed to the following reasons. Firstly, oxygen deficient $\mathrm{WO}_{3-x} \mathrm{NWs}$ directly reduce $\mathrm{Pd}^{2+}$ to metallic Pd NPs with clean surfaces deposited on $\mathrm{WO}_{3-x}$ support; secondly, strong metal-support interaction indeed contributes to the enhanced catalytic performance of supported Pd NPs; additionally, the smaller sized Pd NPs exhibit higher catalytic activities in general.

\section{Conclusions}

In summary, we have developed a novel and simple method to synthesize blue $\mathrm{WO}_{3-x}$ NWs with abundant oxygen vacancies. Notably, palladium NPs directly grow on $\mathrm{WO}_{3-x}$ surfaces through an in situ redox reaction between reductive $\mathrm{WO}_{3-x}$ and 
$\mathrm{Pd}^{2+}$ ions in an aqueous solution. Thanks to this in situ redox deposition without any stabilizing agent or reducing agent, strong metal-support interaction occurs between $\mathrm{Pd}$ and $\mathrm{WO}_{3-x}$. More importantly, the as-prepared $\mathrm{Pd} / \mathrm{WO}_{3-x}$ nanocatalyst shows excellent catalytic activity and good stability for the well-known model reduction of 4-nitrophenol to 4-aminophenol and the hydrogenation of styrene. The rate constant of $\mathrm{Pd} / \mathrm{WO}_{3-x}$ catalyst for 4-NP reduction reaction is $0.045 \mathrm{~s}^{-1}$, and the turnover frequency value for the hydrogenation of styrene reaches as high as $1074.5 \mathrm{~h}^{-1}$, comparable to that of recently reported Pd based catalysts. The significantly improved catalytic activity of the hydrogenation reaction is attributed to the small size of surface-clean Pd NPs and the strong interaction between metal and support. Owing to the synthetic simplicity and excellent properties, present work could provide important inspirations for developing other defect-type materials, and this novel route adopted here could be applied as a new strategy to prepare surface-clean metal nanoparticles loaded on support for various chemical transformations.

\section{Acknowledgements}

The authors acknowledge financial support from the National Natural Science Foundation of China (21271165, 21201141), and the Chinese Universities Scientific Fund (QN2011119, QN2452015424).

\section{Notes and references}

1 C. X. Kronawitter, L. Vayssieres, S. Shen, L. Guo, D. A. Wheeler, J. Z. Zhang, B. R. Antoun and S. S. Mao, Energy Environ. Sci., 2011, 4, 3889-3899.

2 F. Zuo, K. Bozhilov, R. J. Dillon, L. Wang, P. Smith, X. Zhao, C. Bardeen and P. Feng, Angew. Chem., Int. Ed., 2012, 51, 6223-6226.

3 S. Vallejos, T. Stoycheva, P. Umek, C. Navio, R. Snyders, C. Bittencourt, E. Llobet, C. Blackman, S. Moniz and X. Correig, Chem. Commun., 2011, 47, 565-567.

4 A. Tanaka, K. Hashimoto and H. Kominami, J. Am. Chem. Soc., 2014, 136, 586-589.

5 B. J. Liu, J. Zheng, J. L. Wang, J. Xu, H. H. Li and S. H. Yu, Nano Lett., 2013, 13, 3589-3593.

6 F. Liu, L. Li, F. Mo, J. Chen, S. Deng and N. Xu, Cryst. Growth Des., 2010, 10, 5193-5199.

7 H. Zheng, J. Z. Ou, M. S. Strano, R. B. Kaner, A. Mitchell and K. Kalantar-zadeh, Adv. Funct. Mater., 2011, 21, 2175-2196.

8 C. Di Valentin and G. Pacchioni, Acc. Chem. Res., 2014, 47, 3233-3241.

9 F. Lei, Y. Sun, K. Liu, S. Gao, L. Liang, B. Pan and Y. Xie, J. Am. Chem. Soc., 2014, 136, 6826-6829.

10 H. Zhang, C. Huang, R. Tao, Y. Zhao, S. Chen, Z. Sun and Z. Liu, J. Mater. Chem., 2012, 22, 3354-3359.

11 L. Menard, F. Xu, R. Nuzzo and J. Yang, J. Catal., 2006, 243, 64-73.

12 H. Yin, C. Wang, H. Zhu, S. H. Overbury, S. Sun and S. Dai, Chem. Commun., 2008, 4357-4359.

13 X. Pan and Y.-J. Xu, J. Phys. Chem. C, 2013, 117, 17996-18005.
14 C. Hu, X. Zhang, X. Li, Y. Yan, G. Xi, H. Yang and H. Bai, Chem.-Eur. J., 2014, 20, 13557-13560.

15 S. Ida and T. Ishihara, J. Phys. Chem. Lett., 2014, 5, 25332542.

16 C. Yang, Z. Wang, T. Lin, H. Yin, X. Lu, D. Wan, T. Xu, C. Zheng, J. Lin, F. Huang, X. Xie and M. Jiang, J. Am. Chem. Soc., 2013, 135, 17831-17838.

17 X. Pan, M. Q. Yang, X. Fu, N. Zhang and Y. J. Xu, Nanoscale, 2013, 5, 3601-3614.

18 G. Wang, Y. Ling, H. Wang, X. Yang, C. Wang, J. Z. Zhang and Y. Li, Energy Environ. Sci., 2012, 5, 6180-6187.

19 A. M. Smith, M. G. Kast, B. A. Nail, S. Aloni and S. W. Boettcher, J. Mater. Chem. A, 2014, 2, 6121-6129.

20 J. Ding, Y. Chai, Q. Liu, X. Liu, J. Ren and W.-L. Dai, J. Phys. Chem. C, 2016, 120, 4345-4353.

21 K. Nguyen, M. Monteverde, A. Filoramo, L. Goux-Capes, S. Lyonnais, P. Jegou, P. Viel, M. Goffman and J.-P. Bourgoin, Adv. Mater., 2008, 20, 1099-1104.

22 S. Kundu, D. Huitink and H. Liang, Colloids Surf., A, 2010, 360, 129-136.

23 J. F. Moulder, W. F. Stickle, P. E. Sobol and K. D. Bomben, Handbook of X-ray Photoelectron Spectroscopy, 1979, p. 55.

24 J. H. Kang, E. W. Shin, W. J. Kim, J. D. Park and S. H. Moon, J. Catal., 2002, 208, 310-320.

25 G. Wang, H. Wang, Y. Ling, Y. Tang, X. Yang, R. C. Fitzmorris, C. Wang, J. Z. Zhang and Y. Li, Nano Lett., 2011, 11, 3026-3033.

26 G. Xi, S. Ouyang, P. Li, J. Ye, Q. Ma, N. Su, H. Bai and C. Wang, Angew. Chem., Int. Ed., 2012, 51, 2395-2399.

27 Y. H. Li, P. F. Liu, L. F. Pan, H. F. Wang, Z. Z. Yang, L. R. Zheng, P. Hu, H. J. Zhao, L. Gu and H. G. Yang, Nat. Commun., 2015, 6, 8064.

28 F. Liu, X. Chen, Q. Xia, L. Tian and X. Chen, RSC Adv., 2015, 5, 77423-77428.

29 S. Rahimnejad, J. Hui He, F. Pan, X. e. Lee, W. Chen, K. Wu and G. Qin Xu, Mater. Res. Express, 2014, 1, 045044.

30 R. Gazzinelli and O. F. Schirmer, J. Phys. C: Solid State Phys., 1977, 10, 145-149.

31 R. R. Rakhimov, D. E. Jones, H. L. Rocha, A. I. Prokof'ev and A. I. Aleksandrov, J. Phys. Chem. B, 2000, 104, 10973-10977.

32 S. Saha, A. Pal, S. Kundu, S. Basu and T. Pal, Langmuir, 2010, 26, 2885-2893.

33 L. Zhou, Z. Liu, H. Zhang, S. Cheng, L. J. Fan and W. Ma, Nanoscale, 2014, 6, 12971-12980.

34 H. F. Zarick, W. R. Erwin, J. Aufrecht, A. Coppola, B. R. Rogers, C. L. Pint and R. Bardhan, J. Mater. Chem. A, 2014, 2, 7088-7098.

35 J. Zeng, Q. Zhang, J. Chen and Y. Xia, Nano Lett., 2010, 10, 30-35.

36 T. Yao, T. Cui, X. Fang, F. Cui and J. Wu, Nanoscale, 2013, 5, 5896-5904.

37 X. Gu, W. Qi, X. Xu, Z. Sun, L. Zhang, W. Liu, X. Pan and D. Su, Nanoscale, 2014, 6, 6609-6616.

38 Z. Dong, X. Le, Y. Liu, C. Dong and J. Ma, J. Mater. Chem. A, 2014, 2, 18775-18785.

39 J. Sun, Y. Fu, G. He, X. Sun and X. Wang, Catal. Sci. Technol., 2014, 4, 1742-1748. 
40 S. Gao, W. Li and R. Cao, J. Colloid Interface Sci., 2015, 441, 42 F. A. Harraz, S. E. El-Hout, H. M. Killa and I. A. Ibrahim, J. 85-89.

Catal., 2012, 286, 184-192.

41 Y. Niu, L. K. Yeung and R. M. Crooks, J. Am. Chem. Soc., 2001, 43 Y. Pan, D. Ma, H. Liu, H. Wu, D. He and Y. Li, J. Mater. Chem., 123, 6840-6846. 2012, 22, 10834-10839. 